\title{
Muon to electron conversion search in the presence of Al nuclei at the Fermilab Mu2e experiment: Motivation, Design and Progress
}

\author{
Mete Yucel ${ }^{1, *}$ \\ Fermi National Accelerator Laboratory, \\ Batavia, IL, USA \\ E-mail: myucel@fnal.gov
}

The Mu2e experiment aims to find charged lepton flavor violation(CLFV) by measuring the monochromatic electrons from $\mu^{-} N \rightarrow e^{-} N$ conversion with an unprecedented single event sensitivity of $3 \times 10^{-17}$. When completed, the experiment will improve the current limit by four orders of magnitude and make a previously unexplored phase space available for the search for beyond the standard model physics. Physics motivation for the experiment is discussed and design choices for the detectors and various sub systems are explained in this document. The construction of the Mu2e experiment is well underway and commissioning will begin in late 2022 with first data taking in late 2025 and 4-5 years of data taking thereafter to reach our goal.

40th International Conference on High Energy physics - ICHEP2020

July 28 - August 6, 2020

Prague, Czech Republic (virtual meeting)

${ }^{1}$ on behalf of the Mu2e Collaboration[1]

* Speaker 


\section{Motivation}

Charged lepton flavor violation (CLFV) remains one of the most promising processes to find a beyond standard model signal [2] and various experiments around the world are currently developing detectors in order to observe it. Branching ratios for processes like $\mu^{-} N \rightarrow e^{-} N, \mu^{+} \rightarrow e^{+} \gamma, \mu^{+} \rightarrow$ $e^{+} e^{-} e+$ are highly suppressed in the standard model, therefore making any observation a discovery of new physics. With this motivation in mind, the Fermilab based Mu2e experiment aims to find CLFV signal by measuring the monochromatic electrons from $\mu^{-} N \rightarrow e^{-} N$ conversion with an unprecedented single event sensitivity of $3 \times 10^{-17}$. Intense proton beam delivered to the Fermilab Muon campus allows for high statistics when using the right target to produce muons compared to collider experiments with broader focus. Mu2e will either discover muon to electron conversion or improve the current limit[3] on the CLFV by four orders of magnitude and make a previously unexplored phase space available for the search for beyond the standard model physics.

\section{Design}

The goal of the Mu2e experiment is to find muon-to-electron conversion via observation of the monochromatic $105 \mathrm{MeV}$ conversion electrons. The first step in achieving this is the delivery of a high intensity proton beam to Mu2e production target from Fermilab's delivery ring. The $8 \mathrm{GeV}$ pulsed proton beam is delivered to a $\mathrm{W}$ production target at $1695 \mathrm{~ns}$ intervals to produce muons in the graded magnetic field(2.5-4.6 T) of the Production Solenoid(PS). The PS steers these slow muons to the Transfer Solenoid(TS), which selects low energy and negatively charged particles with the help of its S-shaped geometry and central collimator. Beam is then delivered to the Detector Solenoid that houses the stopping target, the tracker and the calorimeter. The general layout of the experiment can be seen in Figure 1.

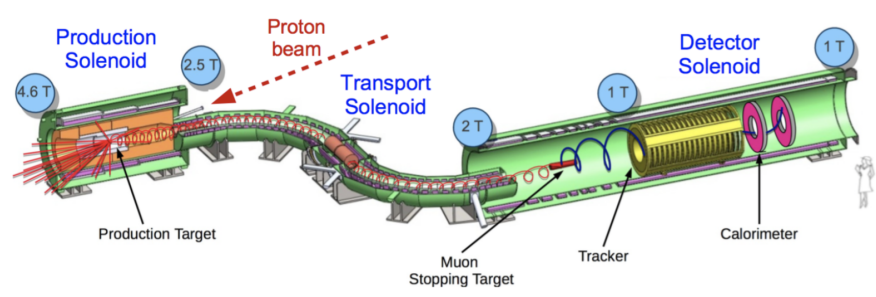

Figure 1: General layout of the Mu2e experiment.

Most of the beam induced backgrounds have relatively short lives compared to the bound muon lifetime. Based on this fact, the pulsed beam structure allows Mu2e experiment to establish a delayed live detection window to capture conversion events with high efficiency as seen in Figure 2. Monochromatic $105 \mathrm{MeV}$ conversion electrons are produced in the presence of stopping target's Al nuclei with a significant decay in orbit (DIO) background that can mimic the conversion electron. Eliminating DIO background requires excellent momentum separation. Therefore, main detector of the Mu2e experiment is a low mass tracking detector with high energy resolution[4]. The Mu2e Tracker is designed to be only sensitive to $105 \mathrm{MeV} / \mathrm{c}$ conversion electron tracks by only covering 


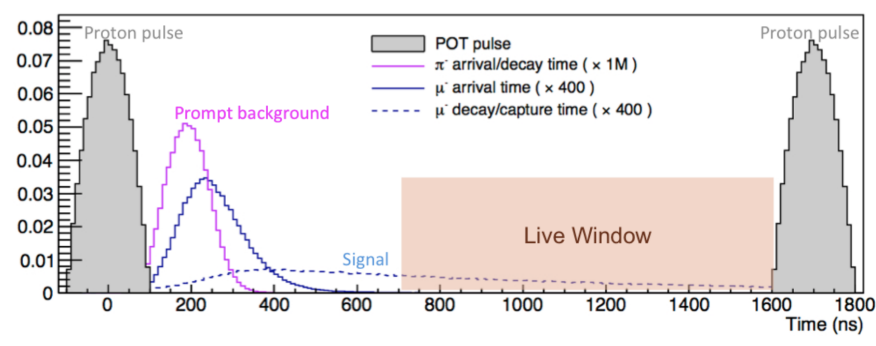

Figure 2: The Mu2e live event window that shows the 700 to $1600 \mathrm{~ns}$ time slot after proton spill where events will be recorded.

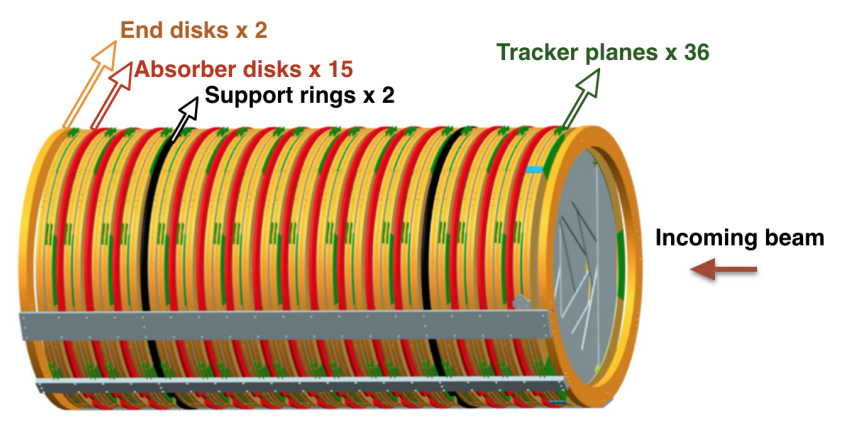

Figure 3: The Mu2e tracker consist of 36 planes placed facing the incoming beam scattering from the stopping target. There are two end disks and support disks to give the detector structural support. In between planes are absorber rings that protect front end electronics placed in tracker planes.

the $700-810 \mathrm{~mm}$ radial region of the cross sectional detector area. Covered area is populated by $216005 \mathrm{~mm}$ diameter low mass Mylar straw drift tubes. Straws are placed in tracker panels in a two layer configuration and 6 of those panels are assembled together to create a tracker plane. The tracker consists of 36 such planes. Signal is read through a $25 \mu \mathrm{m} \mathrm{W}$ wire held at high voltage and positioned at the center of the straw. Each end of the straw is read separately to determine position along the straw and also integrated to measure energy. Signal is processed through the Digital Readout and Control (DRAC) board that is housed inside the detector volume and sent outside via optical link. The Mu2e tracker aims to have better than $180 \mathrm{keV} / \mathrm{c}$ momentum resolution to achieve its targeted DIO background separation. The tracker design can be seen in Figure 3.

The Electromagnetic calorimeter sits directly behind the tracker and offers its own energy and timing measurements to complement the tracker. The calorimeter follows the same design pattern as the tracker, having a hole in the middle to avoid DIO electrons. The calorimeter consists of 1348 CsI crystals arranged in two disks as seen in Figure 4. Disks are separated by $70 \mathrm{~cm}$, the half wavelength of conversion electrons. Silicon photomultipliers are attached at the back of each crystal for the readout. The calorimeter provides $1 \mathrm{~cm}$ spatial resolution and $0.5 \mathrm{~ns}$ timing resolution with $10 \%$ energy resolution for the conversion electrons.

Another unique challenge for Mu2e is the suppression of cosmic background with the inclusion of a cosmic ray veto (CRV) system. Intrinsically, cosmic rays produce 1 conversion-like event per 


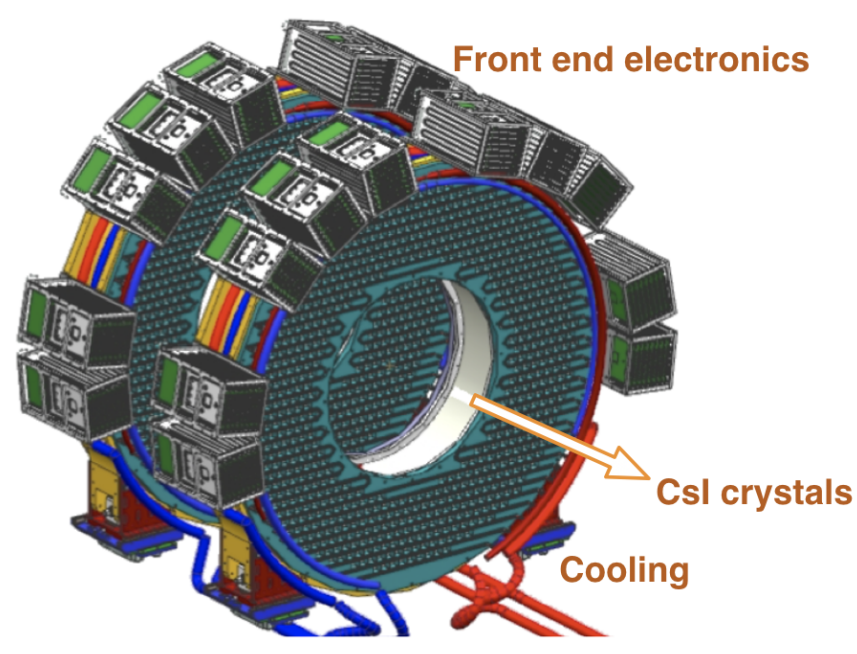

Figure 4: The Mu2e EM calorimeter is made from two disks that are separated by $70 \mathrm{~cm}$. While CsI crystals are arranged in front of the beam, front end electronics are located in crates that attached along the circumference of the detector.

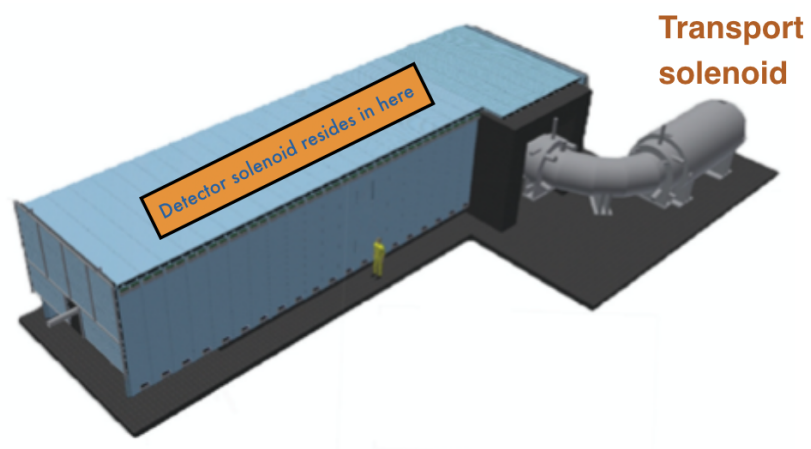

Figure 5: The CRV system is designed to cover all of the detector solenoid as well as half of the transport solenoid to achieve the design goal of having less than one background event for the entirety of the experiment.

day. This background needs to be controlled to achieve sensitivity needed for $\mu^{-} N \rightarrow e^{-} N$ discovery. To achieve such precision, the cosmic ray veto system is designed to encapsulate all of the DS and most of the TS as shown in Figure 5. Polystyrene scintillators coated with $\mathrm{TiO}_{2}$ are sandwiched between $\mathrm{Al}$ absorbers to make di-counters. Signal is transferred via wavelength shifters and readout by silicon photomultipliers. Produced in various cross sections, these di-counters covers a total of $337 \mathrm{~m}^{2}$ area. Each wall is made of 4 layers of di-counters and muons are rejected when they trigger 3 out of 4 layers. The cosmic ray veto system aims to reach $99.99 \%$ veto efficiency during the the life span of the Mu2e experiment to achieve target sensitivity. 


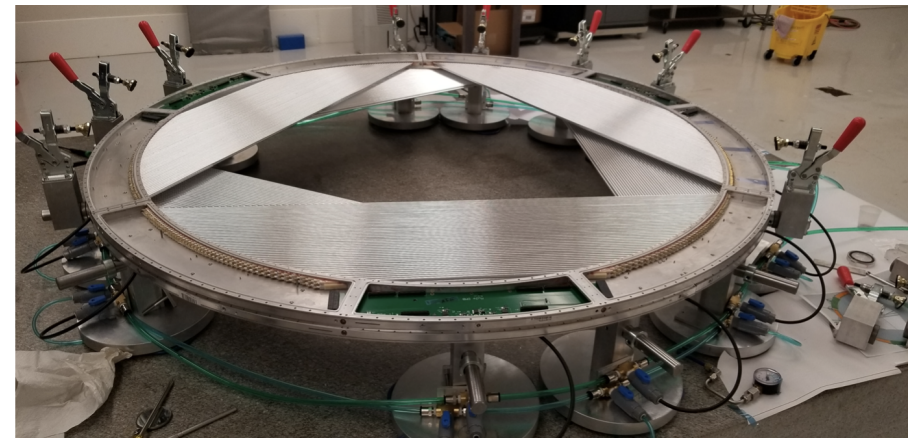

Figure 6: Tracker panels produced are assembled into tracker planes. A plane consists of 6 tracker panels arranged in a two-layered configuration.
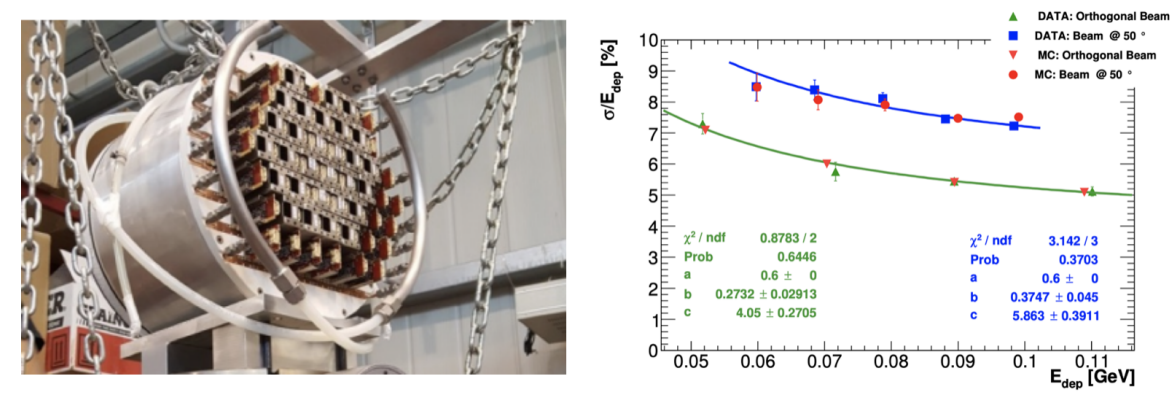

Figure 7: Left: EM calorimeter prototype name Module-0 was tested at the beam test facility of INFN Frascati in 2017. Right: Results from this test showing the energy resolution for Module-0.

\section{Progress}

The Mu2e experiment has over 200 scientists distributed among more than 30 different international institutes. Tremendous progress is still being made through efforts of everybody involved even in these difficult times. One of the most challenging and indispensable parts of the experiment are the solenoids. They are being built and tested under close inspection and supervision of Mu2e scientists and engineers. TS module production is almost finished and expected by the end of 2020 . Similarly PS and DS are also in production and expected to be delivered in 2022.

Tracker panel production is going strong with 40 out of 216 panels produced. Finished panels are configured into tracker planes as shown in Figure 6. The first plane was made in October 2020 and it is planned to be tested throughout next year in a vertical slice test to gain crucial information on the performance of the tracker.

Similarly, the EM Calorimeter already finished testing a prototype made of 51 CsI crystals and the associated readout. The protoype module was able to achieve 5\% energy resolution[5] as shown in Figure 7. Production of CsI crystals is complete and they are planned to be assembled into the disks soon. Progress was also made on the CRV system and 20 out of 83 production modules are completed in 2020. 


\section{Summary}

The Mu2e experiment will improve the current limit on $\mu^{-} N \rightarrow e^{-} N$ conversion rate by four orders of magnitude at $R_{\mu e}=3 \times 10^{-17}$. Only 7 conversion electrons are needed for $5 \sigma$ discovery at $90 \% \mathrm{CL}$. Even without discovery, the experiment will be pushing the explorable mass scale up to $10^{4} \mathrm{TeV}$. Efforts will be focused on finishing the detector elements and commissioning during 2021 and the Mu2e experiment is hoping to reach to $10^{3}$ improvement by 2026 and to $10^{4}$ by the end of the decade.

\section{Acknowledgments}

We are grateful for the vital contributions of the Fermilab staff and the technical staff of the participating institutions. This work was supported by the US Department of Energy; the Istituto Nazionale di Fisica Nucleare, Italy; the Science and Technology Facilities Council, UK; the Ministry of Education and Science, Russian Federation; the National Science Foundation, USA; the Thousand Talents Plan, China; the Helmholtz Association, Germany; and the EU Horizon 2020 Research and Innovation Program under the Marie Sklodowska-Curie Grant Agreement No.690835. This document was prepared by members of the Mu2e Collaboration using the resources of the Fermi National Accelerator Laboratory (Fermilab), a U.S. Department of Energy, Office of Science, HEP User Facility. Fermilab is managed by Fermi Research Alliance, LLC (FRA), acting under Contract No. DE-AC02-07CH11359.

\section{References}

[1] Mu2e Collaboration List, https://mu2e.fnal.gov/mu2e_collaboration_list.shtml 2020

[2] R. Bernstein, et al, Charged Lepton Flavor Violation: An Experimenter's Guide, Phys. Rept. 532(2) 27-64 2013[arXiv:1307.5787]

[3] W. Bertl, et al, A Search for $\mu$ - e conversion in muonic gold, Eur. Phys. J. C 47 337-346 2006

[4] L. Bartoszek, et al, Mu2e Technical Design Report, Fermilab-TM-2594, Fermilab-DESIGN2014-1, 2014 arXiv:1501.05241

[5] N. Atanov, et al, Design and status of the Mu2e crystal calorimeter, IEEE Trans. Nucl. Sci. 65(8) 2073-2080 2018[ arXiv:1802.06346] 